\title{
Optimally Invariant Variable Combinations for Nonlinear Systems
}

\author{
Johannes E. P. Jäschke, Sigurd Skogestad \\ Norwegian University of Science and Technology (NTNU), Trondheim, \\ Norway (e-mail: skoge@chemeng.ntnu.no).
}

\begin{abstract}
In this article we present an "explicit RTO" approach for achieving optimal steady state operation without requiring expensive online calculations. After identifying regions of constant active constraints, it is shown that there exist some optimally invariant variable combination for each region. If the unknown variables can be eliminated by measurements and system equations, the invariant combinations can be used for control. Moreover, we show that the measurement invariants can be used for detecting changes in the active set and for finding the right region to switch to. This explicit RTO approach is applied to a CSTR described by a set of rational equations. We show how the invariant variable combinations are derived, and use polynomial reduction to eliminate the unknown variables to obtain the measurement invariants which are used for control.
\end{abstract}

Keywords: Optimizing control, Polynomial systems, Real-time optimization, Explicit RTO, Self-optimizing control, Optimally invariant measurement combinations, Changing active sets

\section{INTRODUCTION}

Optimal operation of chemical processes becomes increasingly important in order to be able to compete in the international markets and to minimize environmental impact. A well established tool to achieve this goal is realtime optimization (RTO), where the optimal set-points are computed on-line, based on measurements taken at given sample times. This involves setting up and maintaining a real-time computation system, which can be very expensive and time consuming.

An alternative approach is to use off-line calculations and analysis to minimize or avoid complex on-line computations for example by finding optimally invariant measurement combinations ('self-optimizing' variable combinations, (Narasimhan and Skogestad (2007)). Controlling these combinations to their setpoints guarantees to operate the process optimal or close to optimal, with a certain acceptable loss (Skogestad (2000)). The combinations can be controlled by a simple control structure based on PI controllers. The conventional real-time optimization problem can either be replaced completely or partially by controlling invariant variable combinations. In practice, many processes are operated by something similar to this alternative approach, although not always consciously. That is, the optimization problem is not formulated explicitly and the control variables are chosen from experience and engineering intuition.

This publication presents two main results. The first one is extending the idea of self-optimizing control from unconstrained linear problems to constrained nonlinear problems. To the authors knowledge, optimally invariant variable combinations have been considered systematically only for linear plants with quadratic performance index (see e.g. Alstad et al. (2009)). A second contribution is the proof that using controlled variable to identify new sets of active constraints will always identify the correct active set. Although measurement invariants have been used before for active set identification (Manum et al. (2007)), it has not been proved that this holds for nonlinear problems, too.

\section{GENERAL PROCEDURE}

We consider a plant at steady state and assume the plant performance can be modelled as an optimization problem with a performance index $J$ together with equality and inequality constraints, $g(\mathbf{u}, \mathbf{x}, \mathbf{d})$ and $h(\mathbf{u}, \mathbf{x}, \mathbf{d})$ :

$$
\min _{\mathbf{u}, \mathbf{x}} J \quad \text { s.t } \quad\left\{\begin{array}{l}
g(\mathbf{u}, \mathbf{x}, \mathbf{d})=0 \\
h(\mathbf{u}, \mathbf{x}, \mathbf{d}) \leq 0
\end{array}\right.
$$

The variables $\mathbf{u}, \mathbf{x}, \mathbf{d}$ denote the manipulated input variables, the internal states, and the disturbance variables, respectively. In addition, we assume that there are measurements $\mathbf{y}(\mathbf{x}, \mathbf{u}, \mathbf{d})$, which provide information about the internal states and the disturbances of the process.

In order to obtain optimal operation we do not optimize the model on-line at given sample times. Instead, we use the structure of the problem to find optimally invariant variable combinations for the system. Since the available number of degrees of freedom changes when an inequality constraint becomes active, we have to find a new set of invariant measurement combinations for each set of constraints that becomes active during operation of the plant. This makes it necessary to define separate control structures for each region. Therefore, the first step is to partition the operating space into regions defined by the set of active constraints, i.e. the system is optimized for all possible disturbances $\mathbf{d}$ and the active constraints in each region are identified. 
In the second step, we determine (nonlinear) variable combinations which yield optimal operation when kept at their constant setpoint. The variables resulting from this step cannot be used for control directly, because they contain unknown disturbance variables and internal states which are not known. To be able to control the system, we attempt to "model" the variable invariants by expressions which only contain known variables. These can then be used for control in feedback loops.

The last step in this procedure is to define rules for detecting and switching regions when the active constraints change. In many cases this can be done by monitoring the controlled variables of the neighbouring region and switching when the controlled variable of the neighbouring region reaches its optimal value.

\section{DETERMINING INVARIANT VARIABLE COMBINATIONS}

3.1 Invariants for systems with quadratic objective and linear inequality constraints and linear measurements

To illustrate the idea of finding invariant variable combinations we first consider a problem with a quadratic objective and linear constraints. After having identified $n_{r}$ regions of active constraints, we can define an equality constrained optimization problem for each region.

Given $\mathbf{z} \in \mathbb{R}^{n_{z} \times 1}$ and $\mathbf{d} \in \mathbb{R}^{n_{d} \times 1}$, consider the constrained optimization problem:

$$
\min _{\mathbf{z}} J=\min _{\mathbf{z}}\left[\mathbf{z}^{\mathbf{T}} \mathbf{d}^{\mathbf{T}}\right]\left[\begin{array}{cc}
\mathbf{J}_{\mathbf{z z}} & \mathbf{J}_{\mathbf{z d}} \\
\mathbf{J}_{\mathbf{z d}} \mathbf{T} & \mathbf{J}_{\mathbf{d d}}
\end{array}\right]\left[\begin{array}{l}
\mathbf{z} \\
\mathbf{d}
\end{array}\right]
$$

subject to

$$
\mathbf{A}_{z} \mathbf{z}+\mathbf{A}_{d} \mathbf{d}=\tilde{\mathbf{A}}\left[\begin{array}{l}
\mathbf{z} \\
\mathbf{d}
\end{array}\right]=0
$$

where we have $\mathbf{A}_{z} \in \mathbb{R}^{n_{c} \times n_{z}}$ has rank $n_{c}, \mathbf{A}_{d} \in \mathbb{R}^{n_{c} \times n_{d}}$, $\tilde{\mathbf{A}}=\left[\mathbf{A}_{z} \mathbf{A}_{d}\right]$, and $\mathbf{J}_{\mathbf{z z}}>0$.

Eq. (3) may include the model equations as well as active (equality) constraints. Instead of using (3) to eliminate $n_{c}$ internal states to obtain an unconstrained problem, we keep the constraints explicit in the formulation as this more general formulation will be used later when presenting the nonlinear case (where the internal states are not easily substituted). The Karush-Kuhn-Tucker first order optimality conditions give

$$
\nabla_{z} L=\nabla_{z} J+\mathbf{A}_{z}^{\mathbf{T}} \lambda=\tilde{J}\left[\begin{array}{l}
\mathbf{z} \\
\mathbf{d}
\end{array}\right]+\mathbf{A}_{z}^{\mathbf{T}} \lambda=0,
$$

where $\tilde{J}=\left[\mathbf{J}_{\mathbf{u u}} \mathbf{J}_{\mathbf{u d}}\right]$, and $\lambda$ is the vector of Lagrangian multipliers. Therefore, from (4) we have that

$$
\mathbf{A}_{z}^{\mathbf{T}} \lambda=-\tilde{J}\left[\begin{array}{l}
\mathbf{z} \\
\mathbf{d}
\end{array}\right] .
$$

$\mathbf{A}_{z}$ is not full column rank, so let $\mathbf{N}_{z}$ be a basis for the null space of $\mathbf{A}_{z}$ with dimension $n_{D O F}=n_{z}-n_{c}$. Then $\mathbf{N}_{z}^{\mathbf{T}} \mathbf{A}_{z}^{\mathbf{T}}=0$, and at the optimum we must have

$$
\mathbf{c}^{v}(\mathbf{z}, \mathbf{d}) \triangleq \mathbf{N}_{z}^{\mathbf{T}} \tilde{J}\left[\begin{array}{l}
\mathbf{z} \\
\mathbf{d}
\end{array}\right]=0
$$

for the system (5) to be uniquely solvable for $\lambda$. Keeping $\mathbf{c}^{v}(\mathbf{z}, \mathbf{d})$ at zero (in addition to the active constraints), is always optimal. However, it cannot be used for control directly, as it contains unknown (unmeasured) variables. For control, we need a function of measurements $\mathbf{c}(\mathbf{y})$, such that the difference between the invariant and the measurement combination is minimal. Here, we want to "model" $\mathbf{c}^{v}(\mathbf{z}, \mathbf{d})$ perfectly, such that

$$
\mathbf{c}(\mathbf{y})=\mathbf{c}^{v}(\mathbf{z}, \mathbf{d}) \text {. }
$$

Then controlling $\mathbf{c}(\mathbf{y})=0$ yields optimal operation. If we have $n_{z}+n_{d}$ independent linear measurements

$$
y=\mathbf{G}^{y}\left[\begin{array}{l}
\mathbf{z} \\
\mathbf{d}
\end{array}\right],
$$

where $\mathbf{G}^{y}$ is invertible, we can use them with (6) to give

$$
\mathbf{c}(\mathbf{y})=\mathbf{N}^{\mathbf{T}} \tilde{J}\left[\mathbf{G}^{y}\right]^{-1} \mathbf{y} .
$$

However, note that we actually only need $n_{z}-n_{c}+$ $n_{d}=n_{D O F}+n_{d}$ measurements, since the model equations (3) can be used to eliminate the constrained degrees of freedom (internal states). This is shown in Appendix A.

Remark 1. In the unconstrained case, the optimal invariant variable combination is simply the gradient, such that we have $\mathbf{c}(\mathbf{y})=\mathbf{H y}=\nabla_{u} J$, and $\mathbf{H}=\tilde{J}\left[\tilde{\mathbf{G}}^{y}\right]^{-1}$.

\subsection{Invariants for polynomial and rational systems}

An analog approach may be taken for obtaining invariant variable combinations for more general systems described by polynomials. Since rational equations can be transformed into polynomials by multiplying with the common denominator, the method is applicable to rational systems, too.

Initially, all regions defined by constant active constraints are determined. For each region we then have:

Theorem 1. (Nonlinear invariants). Given $\mathbf{z}, \mathbf{d}$ as in section 3.1, consider the nonlinear optimization problem

$$
\min _{\mathbf{z}} J(\mathbf{z}, \mathbf{d}) \quad \text { s.t } \quad g_{i}(\mathbf{z}, \mathbf{d})=0, \quad i=1 \ldots n_{g},
$$

and implicit measurement relations

$$
m_{j}(\mathbf{y}, \mathbf{z}, \mathbf{d})=0 \quad j=1 \ldots n_{y},
$$

where $\mathbf{y}$ is the measured variable. If the Jacobian $\mathbf{A}_{z}(\mathbf{z}, \mathbf{d})=\left[\nabla_{z} g\right]$ has full rank $n_{g}$ at the optimum throughout the region, following holds:

(1) There exist $n_{D O F}=n_{z}-n_{g}$ independent invariant variable combinations $\mathbf{c}^{v}$ with

$$
\mathbf{c}^{v}=\left[\mathbf{N}_{z}(\mathbf{z}, \mathbf{d})\right]^{\mathbf{T}} \nabla_{z} J(\mathbf{z}, \mathbf{d}),
$$

where $\mathbf{N}_{z}(\mathbf{z}, \mathbf{d})$ denotes the null space of the Jacobian of the active constraints $g(\mathbf{z}, \mathbf{d})$.

(2) If there exist polynomials $\alpha_{i}(\mathbf{z}, \mathbf{d})$ and $\beta_{j}(\mathbf{z}, \mathbf{d})$, such that element of $\mathbf{c}^{v}$ can be expressed by

$$
c^{v}=\sum_{i, j}\left(\alpha_{i}(\mathbf{z}, \mathbf{d}) g_{i}(\mathbf{z}, \mathbf{d})+\beta_{j}(\mathbf{z}, \mathbf{d}) m_{j}(\mathbf{y}, \mathbf{z}, \mathbf{d})\right)+c(\mathbf{y}),
$$

then the term $c(\mathbf{y})$ is the desired self-optimizing variable which when controlled to zero yields optimal operation.

Proof. Calculate the Jacobian of the constraints:

$$
\mathbf{A}_{z}(\mathbf{z}, \mathbf{d})=\left[\left[\nabla_{z} g_{1}(\mathbf{z}, \mathbf{d})\right]^{\mathbf{T}}, \cdots,\left[\nabla_{z} g_{n_{g}}(\mathbf{z}, \mathbf{d})\right]^{\mathbf{T}}\right]^{\mathbf{T}}
$$


Since $\mathbf{A}_{z}(\mathbf{z}, \mathbf{d})$ has full rank, the null space has a constant dimension and there exist a unique vector $\lambda$ which satisfies the KKT conditions (Nocedal and Wright (2006)):

$$
\begin{gathered}
\nabla_{z} J(\mathbf{z}, \mathbf{d})+\left[\mathbf{A}_{z}(\mathbf{z}, \mathbf{d})\right]^{\mathbf{T}} \lambda=0 \\
g_{i}(\mathbf{z}, \mathbf{d})=0, \quad i=1 \ldots n_{g}
\end{gathered}
$$

For the existence and uniqueness of $\lambda$, we always must have, that

$$
\left[\mathbf{N}_{z}(\mathbf{z}, \mathbf{d})\right]^{\mathbf{T}} \nabla_{z} J=-\left[\mathbf{N}_{z}(\mathbf{z}, \mathbf{d})\right]^{\mathbf{T}}[\mathbf{A}(\mathbf{z}, \mathbf{d})]^{\mathbf{T}} \lambda,
$$

where $\mathbf{N}_{z}(\mathbf{z}, \mathbf{d})$ is chosen as a basis for the $n_{z}-n_{g^{-}}$ dimensional null space of $\mathbf{A}_{z}(\mathbf{z}, \mathbf{d})$. The optimal invariant variable combination to be kept at $\mathbf{c}^{v}=0$ is then given by:

$$
\mathbf{c}^{v}=\left[\mathbf{N}_{z}(\mathbf{z}, \mathbf{d})\right]^{\mathbf{T}} \nabla_{z} J(\mathbf{z}, \mathbf{d})
$$

The second statement follows from the implicit relations $g_{i}(\mathbf{z}, \mathbf{d})=0$ and $m_{j}(\mathbf{y}, \mathbf{z}, \mathbf{d})=0$.

Remark 2. This variable combination (17) is not unique in the sense that it can be premultiplied by any nonsingular matrix while still yielding optimal operation. However, since the variable combination is derived from the KKT conditions, it is unique in the sense that for a convex optimization problem $\mathbf{c}^{v}=0$ if the system is operated optimally, because the stationary point is unique.

Remark 3. The full rank assumption for $\mathbf{A}_{z}(\mathbf{z}, \mathbf{d})$ at the optimum is valid in most practical cases, as rank deficiency at the optimum implies that the degrees of freedom in the problem change.

Remark 4. The functions $\alpha$ and $\beta$ can be found using Gröbner theory (Cox et al. (1992)), and the condition for the existence of polynomial functions $\alpha_{i}$ and $\beta_{i}$ is that every variable to be eliminated must appear in the initial ideal generated by all $g_{i}$ and $m_{j}$ in lower or equal degree than in $\mathbf{c}^{v}$

To illustrate the concept, we present a toy example.

Example 1. (Nonlinear invariants). We consider a cost function $J\left(z_{1}, z_{2}, d\right)=z_{1}^{2}+z_{2}^{2}$ subject to the constraint

$$
g=\left(z_{1}-1\right)^{2}+\left(z_{2}-d\right)^{2}-5 .
$$

In addition to the known $z_{1}, z_{2}$, the system has one measurement $y$ with the measurement relation

$$
m=z_{1} z_{2}+z_{1} d-y=0 .
$$

First we calculate the Jacobian of (18) with respect to $z$

$$
\mathbf{A}\left(z_{1}, z_{2}, d\right)=\left[2\left(z_{1}-1\right) 2\left(z_{2}-d\right)\right],
$$

and the basis of its null space:

$$
\mathbf{N}\left(z_{1}, z_{2}, d\right)=\left[-\left(z_{2}-d\right)\left(z_{1}-1\right)\right]^{\mathbf{T}} .
$$

After computing the gradient of the cost function $J$

$$
\nabla J=\left[\begin{array}{ll}
2 z_{1} & 2 z_{2}
\end{array}\right]^{\mathbf{T}},
$$

we obtain the invariant variable combination as in (17):

$$
c^{v}=\underbrace{\left[-\left(z_{2}-d\right)\left(z_{1}-1\right)\right]}_{[\mathbf{N}(\mathbf{z}, \mathbf{d})]^{\mathbf{T}}} \underbrace{\left[\begin{array}{c}
2 z_{1} \\
2 z_{2}
\end{array}\right]}_{\nabla J}=2\left(z_{1} d-z_{2}\right)
$$

However, $c^{v}$ contains the unmeasured disturbance $d$, so it cannot be used for control. Using the measurement relation (19) and equation (13) we see by inspection that $\alpha=0$ and $\beta=2$ yield a $c(y)$ which satisfies (7):

$$
\begin{aligned}
c^{v}=\underbrace{2\left(z_{1} d-z_{2}\right)}_{c^{v}} & =\underbrace{0}_{\alpha} g+\underbrace{2}_{\beta} \underbrace{\left(z_{1} z_{2}+z_{1} d-y\right)}_{m} \\
& +\underbrace{2 y-2 z_{2}-2 z_{1} z_{2}}_{\mathbf{c}(\mathbf{y})} .
\end{aligned}
$$

Since $m=0$, we have that $c^{v}=c(y)$. In more complex cases, $\alpha$ and $\beta$ have to be determined by computing a Gröbner basis for the constraint and measurement relations and by reducing $c^{v}$ modulo the Gröbner basis.

\section{SWITCHING OPERATING REGIONS}

After the controlled variables for all regions are identified, the remaining issue is to determine how to switch between operating regions. Under certain assumptions, the switching points can be found using the already defined invariant variable combinations.

Theorem 2. (Switching regions). Assume the system (10) convex and at the optimum $\nabla_{z} J(\mathbf{z}, \mathbf{d}) \neq 0$ wherever a constraint is active. If a disturbance moves the system continuously from one region of active constraints to another, (i.e. the system does not jump over regions) the exact switching points can be detected by monitoring the controlled variables and the constraints of the neighbouring regions.

Proof. We consider to type of changes, denoted type $I$ and $I I$. In changes of type $I$, a constraint is replaced or added to the current active set. This change is easily detected by monitoring the active constraints of the neighbouring regions. As the system is operated optimally and the disturbance moves the system gradually to the new region, the region boundary is reached, when the constraint is hit.

In changes of type $I I$, a constraint becomes inactive and the released degree of freedom is controlled using a measurement invariant. Detecting a type $I I$ change is done by monitoring the invariant variable combinations of the neighbouring regions. If if an invariant variable combination hits the zero value, the region is switched. The invariant variable combinations assume the value zero only at the switching points. This can be seen by contradiction. Consider two regions with $c_{1}^{v}$ and $c_{2}^{v}$. Let the system be operated optimally at $\left(\mathbf{z}_{0}, \mathbf{d}_{0}\right)$ in the constrained region 1 $\left(c_{1}^{v}\left(\mathbf{z}_{0}, \mathbf{d}_{0}\right)=0\right)$, and let $c_{2}^{v}\left(\mathbf{z}_{0}, \mathbf{d}_{0}\right)=0$. The Jacobians of the set of constraints $g^{1}(\mathbf{z}, \mathbf{d})$ and $g^{2}(\mathbf{z}, \mathbf{d})$ are denoted as $\mathbf{A}^{1}(\mathbf{z}, \mathbf{d})$ and $\mathbf{A}^{2}(\mathbf{z}, \mathbf{d})$.

Since $c_{1}^{v}\left(\mathbf{z}_{0}, \mathbf{d}_{0}\right)=c_{2}^{v}\left(\mathbf{z}_{0}, \mathbf{d}_{0}\right)=0$ we have

$$
\underbrace{\left[\mathbf{N}^{1}\left(\mathbf{z}_{0}, \mathbf{d}_{0}\right)\right]^{\mathbf{T}} \nabla_{z} J\left(\mathbf{z}_{0}, \mathbf{d}_{0}\right)}_{=0}=\underbrace{\left[\mathbf{N}^{2}\left(\mathbf{z}_{0}, \mathbf{d}_{0}\right)\right]^{\mathbf{T}} \nabla_{z} J\left(\mathbf{z}_{0}, \mathbf{d}_{0}\right)}_{=0} \text {. }
$$

Since $\nabla_{z} J(\mathbf{z}, \mathbf{d}) \neq 0$, this implies that $\mathbf{A}^{1}$ and $\mathbf{A}^{2}$ are row equivalent and the null spaces of $\mathbf{A}^{1}\left(\mathbf{z}_{0}, \mathbf{d}_{0}\right)$ and $\mathbf{A}^{2}\left(\mathbf{z}_{0}, \mathbf{d}_{0}\right)$ have the same basis. However, this is not possible, because by assumption, $\mathbf{A}^{1}$ and $\mathbf{A}^{2}$ have different ranks. Therefore the invariant variable combination of a "less constrained" region cannot become zero in a region which is "more constrained". Thus, the active constraints and the measurement combinations can be used for determining when to switch region. 


\section{A}

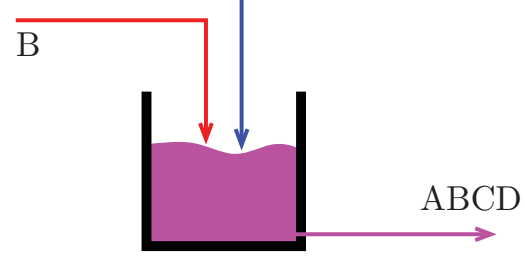

Fig. 1. CSTR with two reactions

Table 1. Variables relevant for control

\begin{tabular}{lc}
\hline Measurements $\mathbf{y}$ & $F, c_{B}, q$ \\
Manipulated variables $\mathbf{u}$ & $F_{A}, F_{B}$ \\
Unknown disturbance d & Rate constant $k_{1}$ \\
Internal states $\mathbf{z}_{\text {unknown }}$ & $c_{A}, c_{C}$ \\
\hline
\end{tabular}

\section{APPLICATION}

We consider an isothermal CSTR with two parallel reactions, Fig. 1, from Srinivasan et al. (2008). Two feed streams $F_{A}$ and $F_{B}$ with the concentrations $c_{A}$ and $c_{B}$ react in a tank to the desired product $C$ and the undesired side product $D$. The tank is equipped with one outflow in which all components are present. In order to enable isothermal reaction conditions a temperature loop is closed such that the correct amount of heat is removed from the system. The temperature control is assumed to be perfect. The products $C$ and $D$ are formed by the reactions:

$$
\begin{array}{cll}
\mathrm{A}+\mathrm{B} & \stackrel{k_{1}}{\longrightarrow} & \mathrm{C} \\
2 \mathrm{~B} & \stackrel{k_{2}}{\longrightarrow} & \mathrm{D}
\end{array}
$$

The optimization objective is to maximize the desired product $\left(F_{A}+F_{B}\right) c_{C}$ weighted by a yield factor $\left(F_{A}+\right.$ $\left.F_{B}\right) c_{C} /\left(F_{A} c_{A, i n}\right)$. The amount of heat to remove and the maximum flow rate are limited. This lets us formulate the optimization problem of the system as follows:

subject to

$$
\max _{F_{A}, F_{B}} \frac{\left(F_{A}+F_{B}\right) c_{C}}{F_{A} c_{A_{i n}}}\left(F_{A}+F_{B}\right) c_{C}
$$

$$
\begin{aligned}
F_{A} c_{A_{i n}}-\left(F_{A}+F_{B}\right) c_{A}-k_{1} c_{A} c_{B} V & =0 \\
F_{B} c_{B_{i n}}-\left(F_{A}+F_{B}\right) c_{B}-k_{1} c_{A} c_{B} V-2 k_{2} c_{B}^{2} V & =0 \\
-\left(F_{A}+F_{B}\right) c_{C}+k_{1} c_{A} c_{B} V & =0 \\
F_{A}+F_{B}-F & =0 \\
k_{1} c_{A} c_{B} V\left(-\Delta H_{1}\right)+2 k_{2} c_{B} V\left(-\Delta H_{2}\right)-q & =0 \\
q-q_{\max } & \leq 0 \\
F-F_{\max } & \leq 0
\end{aligned}
$$

The variables $k_{1}$ and $k_{2}$ are the rate constants for the two reactions, $\left(-\Delta H_{1}\right)$ and $\left(-\Delta H_{2}\right)$ are the corresponding reaction enthalpies, $q$ the heat produced by the reactions, $V$ the reactor volume, and $F$ the total flow rate. The measured variables $(\mathbf{y})$, the manipulated variables $(\mathbf{u})$, the disturbance variables (d), and the internal states are listed in table 1 , and the parameter values of the system are given in table 2 . The combined vector of states and manipulated variables is

$$
\mathbf{z}=\left[c_{A}, c_{B}, c_{C}, F_{A}, F_{B}\right]^{\mathbf{T}} .
$$

\subsection{Identifying operational regions}

The first step of the procedure, optimizing the system offline for all possible values shows that the system operation
Table 2. Parameters

\begin{tabular}{llc}
\hline$k_{1}$ & $\mathrm{l} /(\mathrm{mol} \mathrm{h})$ & $0.3-1.5$ \\
$k_{2}$ & $\mathrm{l} /(\mathrm{mol} \mathrm{h})$ & 0.0014 \\
$\left(-\Delta H_{1}\right)$ & $\mathrm{j} / \mathrm{mol}$ & $7 \times 10^{4}$ \\
$\left(-\Delta H_{2}\right)$ & $\mathrm{j} / \mathrm{mol}$ & $5 \times 10^{4}$ \\
$c_{A, i n}$ & $\mathrm{~mol} / \mathrm{l}$ & 2 \\
$c_{B, i n}$ & $\mathrm{~mol} / \mathrm{l}$ & 1.5 \\
$V$ & $\mathrm{l}$ & 500 \\
$F_{\max }$ & $\mathrm{l}$ & 22 \\
$q_{\max }$ & $\mathrm{kJ} / \mathrm{h}$ & 1000 \\
\hline
\end{tabular}

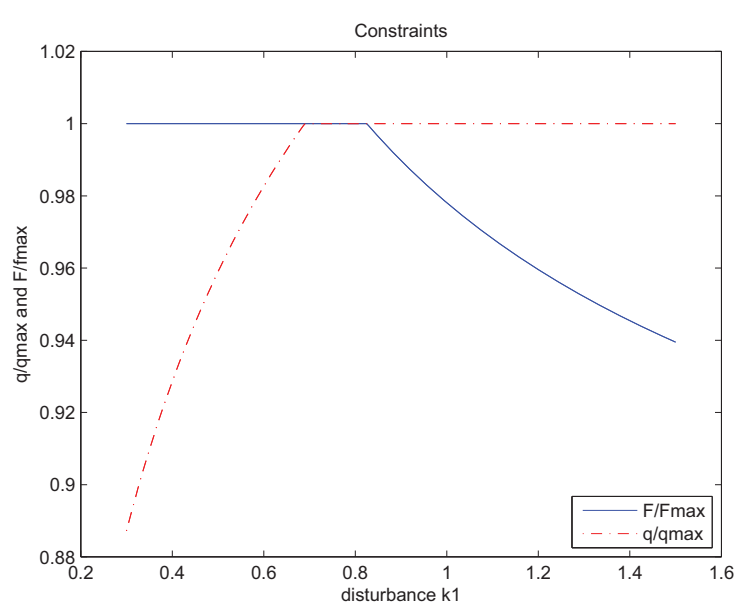

Fig. 2. Optimal values of the constrained variables

space can be partitioned into three regions defined by the set of active constraints. In region 1 , for values of $k_{1}$ below about $k_{1}=0.65$ only the flow constraint is active (Fig. 2). In region 2 for values between $k_{1}=0.65$ and $k_{1}=0.8$ both constraints are active, and in region 3 above $k_{1}=0.8$ only the heat constraint is active.

After satisfying the active constraints in the regions we are left with $N_{\mathrm{DOF}, 1}=1$ for region $1, N_{\mathrm{DOF}, 2}=0$ for region 2 , and $N_{\mathrm{DOF}, 1}=1$ for region 3 .

In region 1 , one of the manipulated variables (flow rates) is used to control the active constraint (maximum flow) and the other manipulated variable is used to control the invariant measurement combination of the region. In region 2 we simply control the active constraints, keeping $q$ at $q_{\max }$ and $F$ at $F_{\max }$. In region 3 , again one of the manipulated variables is used to control the active constraint (maximum heat removal) and the other one is used to control the invariant measurement combination of region 3 .

\subsection{Determining the invariant variable combinations}

Using the information from the previous section, we determine the invariant variable combinations in each region. First, we calculate the null space of Jacobian of the active set $\mathbf{N}_{z}^{\mathbf{T}}$ and multiply it with the gradient of the objective function $\nabla_{z} J(\mathbf{z}, \mathbf{d})$, as in (17) to obtain the invariant variable combination. Generally this is a fractional expression, but since we are controlling it to zero, it is sufficient to consider only the numerator of $\mathbf{N}_{z}^{\mathbf{T}} \nabla_{z} J$. For region 1 we obtain the invariant 


$$
\begin{aligned}
\mathbf{c}_{1}^{v}(\mathbf{z}, \mathbf{d}) & =-\left(F_{A}+F_{B}\right)^{2} c_{C}\left[-3 c_{C} F_{B}^{2} F_{A}-3 c_{C} F_{A}^{2} F_{B}\right. \\
& -4 c_{C} c_{B} F_{A}^{2} k_{2} V-4 c_{C} k_{2} V^{2} k_{1} c_{B}^{2} F_{A}-c_{C} F_{A}^{3} \\
& -c_{C} F_{B}^{3}-4 c_{C} k_{2} V^{2} k_{1} c_{B}^{2} F_{B}-c_{C} c_{B} F_{A}^{2} k_{1} V \\
& -4 c_{C} c_{B} F_{B}^{2} k_{2} V-c_{C} c_{B} F_{B}^{2} k_{1} V-c_{C} F_{A}^{2} c_{A} k_{1} V \\
& -c_{C} F_{B}^{2} c_{A} k_{1} V-8 c_{C} F_{A} c_{B} F_{B} k_{2} V \\
& -2 c_{C} F_{A} c_{B} F_{B} k_{1} V-2 c_{C} F_{A} F_{B} c_{A} k_{1} V \\
& +8 F_{A} k_{1} V^{2} c_{A, i n} k_{2} c_{B}^{2}+2 F_{A}^{2} k_{1} V c_{B} c_{A, i n} \\
& +2 F_{A} k_{1} V F_{B} c_{B} c_{A, i n}-2 F_{A}^{2} k_{1} V c_{B, i n} c_{A} \\
& \left.-2 F_{A} k_{1} V F_{B} c_{B, i n} c_{A}\right]
\end{aligned}
$$

which should be controlled to zero. This invariant can be simplified somewhat further, since we know that $\left(F_{A}+\right.$ $\left.F_{B}\right)^{2} c_{C} \neq 0$. It is therefore sufficient to control the second term in the square brackets in (30) to zero.

As mentioned above, region 2 does not have any unconstrained degree of freedom, so satisfying all active constraints yields optimal operation. In region 3 we obtain an expression similar to (30) for $\mathbf{c}_{3}^{v}(\mathbf{z}, \mathbf{d})$.

\subsection{Eliminating unknown variables}

The invariant variable combination $\mathbf{c}_{1}^{v}(\mathbf{z}, \mathbf{d})$ and $\mathbf{c}_{3}^{v}(\mathbf{z}, \mathbf{d})$ still contain the unknown and internal variables $k_{1}, c_{a}$ and $c_{C}$, so they cannot be used for feedback control directly. In the next step the unknown variables have to be replaced by expressions in the measured variables, so that this invariant can be used for control. Depending on the type of the system equations, different methods may be applied in this step. The general idea is that we use the measurements together with the equations that are satisfied in the active set to express the invariant. As all equations in this case study are polynomial (rational expressions equal to zero can transformed to polynomials by multiplication with the denominator), we attempt to reduce the invariants modulo the active set with a variable ordering that eliminates the unknowns. To simplify the elimination procedure, $k_{1}$ is eliminated by solving the third equality constraint for $k_{1}$.

$$
k_{1}=\left(F_{A}+F_{B}\right) c_{C} /\left(c_{A} c_{B} V\right)
$$

and inserting it into (30). The other unknown variables $c_{A}$ and $c_{C}$ are eliminated using polynomial reduction and the resulting measurement invariant in region 1 becomes:

$$
\begin{aligned}
c_{1}(\mathbf{y}) & =-F_{\max }\left(F_{\max } c_{B}+2 c_{B}^{2} k_{2} V-F_{B} c_{B, i n}\right)^{2} \\
& \left(4 c_{B}^{4} k_{2}^{2} V^{2}+4 F_{\max } c_{B}^{3} k_{2} V-6 k_{2} V c_{B}^{2} F_{B} c_{A, i n}\right. \\
& -4 k_{2} V F_{\max } c_{B, i n} c_{B}^{2}+6 k_{2} V c_{B}^{2} F_{\max } c_{A, i n} \\
& +F_{\max }^{2} c_{B}^{2}-2 F_{\max }^{2} c_{B, i n} c_{B}+2 c_{B} F_{\max }^{2} c_{A, i n} \\
& -2 c_{B} F_{\max } F_{B} c_{A, i n}-F_{B}^{2} c_{B, \text { in }}^{2}+3 F_{\max } F_{B} c_{A, i n} c_{B, i n} \\
& \left.-F_{B}^{2} c_{A, i n} c_{B, i n}+2 F_{\max } F_{B} c_{B, \text { in }}^{2}-2 F_{\max }^{2} c_{A, i n} c_{B, i n}\right)
\end{aligned}
$$

This expression depends only on known variables and parameters. The measurement invariant for region 3 is found in the same way:

$$
\begin{aligned}
c_{3}(\mathbf{y}) & =-\left(F_{A} c_{B}+c_{B} F_{B}+2 c_{B}^{2} k_{2} V-c_{B, i n} F_{B}\right)^{2} \\
& \left(-3 F_{B}^{2} q_{\max } c_{B, i n} c_{B}+8 c_{B}^{4} q_{\max } k_{2}^{2} V^{2}\right. \\
& +F_{B}^{2} q_{\max } c_{B, i n}^{2}+2 c_{B}^{2} F_{B}^{2} q_{\max }+2 F_{A}^{2} q_{\max } c_{B}^{2} \\
& +4 c_{B}^{4} F_{B} k_{2}^{2} c_{B, i n} V^{2} \Delta H_{2}+8 c_{B}^{3} F_{B} q_{\max } k_{2} V \\
& +2 c_{B}^{3} F_{B}^{2} k_{2} c_{B, i n} V \Delta H_{2}-6 c_{B}^{2} F_{B} q_{\max } k_{2} c_{B, i n} V \\
& -2 c_{B}^{2} F_{B}^{2} k_{2} c_{B, i n}^{2} V \Delta H_{2}-F_{A}^{2} q_{\max } c_{B, i n} c_{B} \\
& +4 F_{A} c_{B}^{2} F_{B} q_{\max }+F_{A} F_{B} q_{\max } c_{B, i n}^{2}+2 F_{A}^{2} c_{B} q_{\max } c_{A, i n} \\
& +6 F_{A}^{2} k_{2} c_{B, i n} V \Delta H_{2} c_{B}^{3}+12 F_{A} k_{2}^{2} c_{B, i n} V^{2} \Delta H_{2} c_{B}^{4} \\
& +8 F_{A} c_{B}^{3} F_{B} k_{2} c_{B, i n} V \Delta H_{2}+8 F_{A} c_{B}^{3} q_{\max } k_{2} V \\
& -2 F_{A} c_{B}^{2} q_{\max } k_{2} c_{B, i n} V-6 F_{A} c_{B}^{2} F_{B} k_{2} c_{B, i n}^{2} V \Delta H_{2} \\
& -4 F_{A} F_{B} q_{\max } c_{B, i n} c_{B}+2 F_{A}^{2} c_{B}^{2} k_{2} c_{A, i n} c_{B, i n} V \Delta H_{2} \\
& -F_{A}^{2} q_{\max } c_{A, i n} c_{B, i n}+4 F_{A}^{2} c_{B}^{3} k_{2} c_{A, i n} V \Delta H_{2} \\
& +4 F_{A} c_{B}^{3} F_{B} k_{2} c_{A, i n} V \Delta H_{2}-2 F_{A} c_{B}^{2} F_{B} k_{2} c_{A, i n} c_{B, i n} V \Delta H_{2} \\
& +4 F_{A} c_{B}^{2} q_{\max } k_{2} c_{A, i n} V+2 F_{A} c_{B} F_{B} q_{\max } c_{A, i n} \\
& \left.-F_{A} F_{B} q_{\max } c_{A, i n} c_{B, i n}\right)
\end{aligned}
$$

Although these expressions seem complicated, they contain only known variables and can therefore be easily evaluated and controlled to their setpoint using a PI controller. In both invariants, the term in the first bracket is never zero (to see this, compare it with the second equality constraint in (28)), so it is sufficient to control the term in the second bracket to zero.

The values of these polynomials vary over order of magnitudes, so they are scaled to avoid numerical problems. The invariant of region 1 was scaled by $10^{5}$ and the invariant of region 3 was scaled by $10^{6}$.

\subsection{Using measurement invariants for control and region identification}

We use the controlled variables of the neighbouring regions for determining when to switch. Starting in region 1 optimal operation is achieved by using the two inputs $F_{A}$ and $F_{B}$ to control $c_{1}(\mathbf{y})=0$ and $F_{A}+F_{B}=F_{\max }$. If $k_{1}$ increases, the amount of heat to be removed (the controlled variable of region 2 ) increases until it reaches the maximum possible value, $q_{\max }$ (Fig 3 ). When this value is reached, the control structure has to be changed to region 2. Now the inputs are used to control $q=q_{\max }$ and $F_{A}+$ $F_{B}=F_{\max }$. While operating in region 2 the controlled variables of the neighbouring regions, $c_{1}(\mathbf{y})$ and $c_{3}(\mathbf{y})$ are monitored. If $k_{1}$ increases further, $c_{3}(\mathbf{y})$ approaches its optimal setpoint for region 3 and we switch region when the optimal value is reached. Switching back from the different regions is done in an analog manner.

\section{DISCUSSION}

The invariant variable combinations above are obtained by a two-step method, in which first the Lagrangian multipliers are eliminated, and subsequently the unknown variables (disturbances and internal states) are replaced by measurement relations. However, for systems which can be described by rational functions, as the CSTR example, there exists possibilities to eliminate both, the Lagrangian multipliers and the unknown variables simultaneously. 


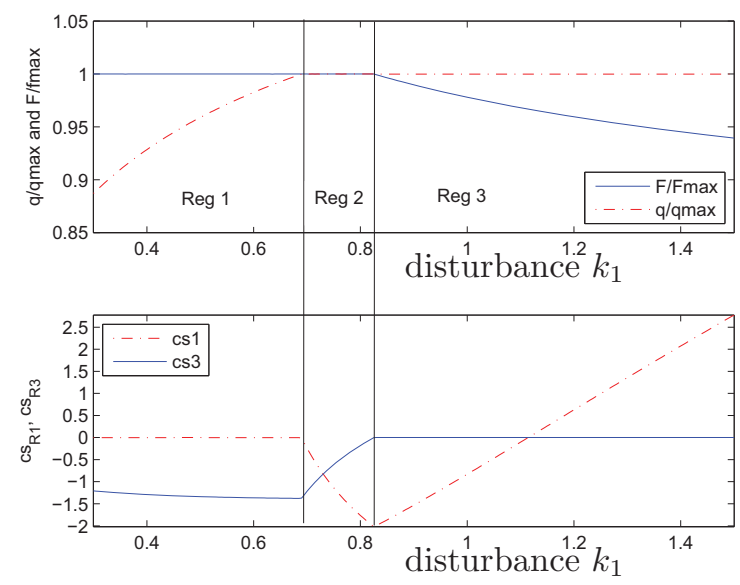

Fig. 3. Optimal values of controlled variables

This is done by defining an ideal which is generated by the polynomials describing the Karush-Kuhn-Tucker conditions and choosing an appropriate term order to eliminate the unknown disturbance and state variables (Cox et al. (1992)) Applying the chosen order to generate the elimination ideal gives a set of equations which are fulfilled at all times when the KKT conditions hold, but which do not contain any of the unknown variables.

Choosing a polynomial from this set which is not a (polynomial) combination of the equality constraints (i.e. which is not in the ideal generated by the equality constraints), gives a candidate for the measurement invariant. However, presently there are two challenges with the simultaneous method. First, the system with the chosen invariant measurement combinations may have (many) more roots than the KKT system. If we use them for control, we might control to "solutions" which do not satisfy the first order optimality conditions. Second, it is generally difficult to determine a term ordering a priori which eliminates the unknown variables and, at the same time ensures that the equations in the elimination ideal are not polynomial combinations of the equality constraints. If this is the case, the chosen variable combination is in the ideal generated by the equality constraints and the invariant will always be zero when the equality constraints are satisfied. This leads to an infinite number of solutions for the system. So far we are not aware of a method to handle these challenges in a systematic way, but we have found invariants in the elimination ideals which yield optimal operation in for the CSTR case study shown above.

The two step method presented in this work fundamentally shows the existence of invariant variable combinations for nonlinear systems and gives an easy way to compute and to use them. Additionally, in contrast to the elimination ideal method, the two-step method is principally not restricted to polynomial or rational models, provided that the unknowns can be eliminated.

\section{CONCLUSION}

The procedure presented in this paper is applicable to nonlinear steady state optimization problems and consists of four steps. First, regions of constant active constraints are defined. Second, optimally invariant nonlinear variable combinations are determined for each of the regions. Third, the unknown internal variables and disturbances are eliminated from the invariants to obtain variable combinations containing only known variables (measurements). It is proven that these variables can be used to uniquely identify a new active set. This makes the method applicable over a wide disturbance range with changing active sets. Finally, we have applied the method to a case study with a four component isothermal CSTR.

Although designing a self-optimizing control structure may require more work in advance, its implementation and maintenance is easy in practice. After the control structure is designed, optimal operation can achieved by simple PI controllers and there is no need to invest in expensive realtime equipment to operate the process optimally.

\section{REFERENCES}

Alstad, V., Skogestad, S., and Hori, E. (2009). Optimal measurement combinations as controlled variables. Journal of Process Control.

Cox, D., Little, J., and O'Shea, D. (1992). Ideals, Varieties, and Algorithms. Springer-Verlag.

Manum, H., Narasimhan, S., and Skogestad, S. (2007). A new approach to explicit mpc using self-optimizing control, Internal report. www.nt.ntnu.no/users/skoge/publications/2007.

Narasimhan, S. and Skogestad, S. (2007). Implementation of optimal operations using off-line computations. 8th international IFAC Symposium on Dynamics and Control of Process Systems (DYCOPS), Cancun, Mexico.

Nocedal, J. and Wright, S.J. (2006). Numerical Optimization. Springer.

Skogestad, S. (2000). Plantwide control: The search for the self-optimizing control structure. Journal of Process Control, 10, 487-507.

Srinivasan, B., Biegler, L.T., and Bonvin, D. (2008). Tracking the necessary conditions of optimality with changing set of active constraints using a barrier-penalty function. Computers and Chemical Engineering, 32, $572-279$.

\section{Appendix A. ELIMINATING INTERNAL STATES}

We define the combined input and internal state vector as

$$
\mathbf{z}=\left[\begin{array}{ll}
\mathbf{x}^{\mathbf{T}} & \mathbf{u}^{\mathbf{T}}
\end{array}\right]^{\mathbf{T}} .
$$

It is assumed that we have $n_{u}+n_{d}$ independent measurements for system 3 .

$$
\mathbf{y}=\tilde{\mathbf{G}}^{y}\left[\mathbf{u}^{\mathbf{T}} \mathbf{d}^{\mathbf{T}}\right]
$$

with $\tilde{\mathbf{G}}^{y}$ invertible. Then

$$
\left[\begin{array}{l}
\mathbf{z} \\
\mathbf{d}
\end{array}\right]=\left[\begin{array}{l}
\mathbf{x} \\
\mathbf{u} \\
\mathbf{d}
\end{array}\right]=\left[\begin{array}{cc}
-\mathbf{A}_{x}^{-1} \mathbf{A}_{u} & -\mathbf{A}_{x}^{-1} \mathbf{A}_{s} \\
\mathbf{I} & 0 \\
0 & \mathbf{I}
\end{array}\right] \underbrace{\left[\begin{array}{c}
\mathbf{u} \\
\mathbf{d}
\end{array}\right]}_{=\left[\tilde{\mathbf{G}}^{y}\right]^{-1} \mathbf{y}},
$$

and we derive the optimal measurement combination which satisfies (7) as

$$
\mathbf{c}(\mathbf{y})=\mathbf{H y}
$$

with

$$
\mathbf{H}=\mathbf{N}_{z}^{\mathbf{T}} \tilde{J}\left[\begin{array}{cc}
-\mathbf{A}_{x}^{-1} \mathbf{A}_{u} & -\mathbf{A}_{x}^{-1} \mathbf{A}_{s} \\
\mathbf{I} & 0 \\
0 & \mathbf{I}
\end{array}\right]\left[\tilde{\mathbf{G}}^{y}\right]^{-1} .
$$

